\title{
A revisit to a low-cost method for the isolation of microsatellite markers: the case of the endangered Malayan tapir (Tapirus indicus)
}

\author{
QI LUAN LIM ${ }^{1}$, NURUL ADILAH ISMAIL ${ }^{1}$, RAMITHA ARUMUGAM ${ }^{1}$, \\ WEI LUN NG ${ }^{2}$, CHRISTINA SEOK YIEN YONG ${ }^{1}$, AHMAD ISMAIL $^{1}$, \\ JEFFRINE J. ROVIE-RYAN ${ }^{3,4}$, NORSYAMIMI ROSLI ${ }^{3}$ and GEETHA ANNAVI ${ }^{1}$
}

\begin{abstract}
There are many approaches to develop microsatellite markers. Despite the availability of the more advanced technology in the market, due to budget constraints, we revisited an easy and rapid polymerase chain reaction (PCR) cloning-sequencing method to design microsatellite markers for Tapirus indicus. Using six random amplified microsatellite markers, this study had rapidly generated 45 unique genomic sequences containing microsatellites. After screening 15 terminal and seven intermediate microsatellite loci, we shortlisted five and seven which were amplified either by single- or multiplex PCR using the economical three-primer PCR method. Genotyping attempts were made with ten T. indicus individuals using three of the terminal microsatellite loci and all seven intermediate loci. However, none of the terminal microsatellite loci were considered useful for population genotyping studies, while the seven intermediate loci showed good amplification but were monomorphic in the ten samples and the subsequent 51 tapir samples. Despite successful detection of amplified loci, we would like to highlight that, researchers who are interested in this alternative method for isolation of microsatellite loci to be cautious and be aware of the limitations and downfalls reported herein that could render these loci unsuitable for population genotyping.
\end{abstract}

Keywords: Tapirus indicus, Malayan tapir, Asian tapir, Random Amplified Microsatellite, microsatellite development, genotyping

\section{INTRODUCTION}

Microsatellites, also known as short tandem repeats (STRs) or short sequence repeats (SSRs), are stretches of DNA consisting of tandemly repeated 1-6 nucleotides occurring at high frequency in the nuclear genomes of most organisms, with the length of a microsatellite locus typically in 5-40 repeats (Selkoe and Toonen, 2006). Slippage event during DNA replication is generally considered as the main mechanism for the expansion and contraction of microsatellites (Moniruzzaman et al., 2015). Because of the hypermutability, microsatellite markers have been shown to be highly polymorphic (Guichoux et al., 2011). They are widely used for molecular genetic studies including fingerprinting, parental or kinship analysis, population genetic structure and biological resources conservation (Crawford et al., 2008; Moniruzzaman et al., 2015; Putman and Carbone, 2014).

\footnotetext{
${ }^{1}$ Department of Biology, Faculty of Science, Universiti Putra Malaysia, Serdang, Selangor, Malaysia

${ }^{2}$ China-ASEAN College of Marine Sciences, Xiamen University Malaysia

${ }^{3}$ National Wildlife Forensic Laboratory (NWFL), Ex-Situ Conservation Division, Department of Wildlife and National Parks (DWNP), Kuala Lumpur, Malaysia

${ }^{4}$ Institute of Tropical Biodiversity and Sustainable Development, Universiti Malaysia Terengganu, Kuala Terengganu, Terengganu, Malaysia

Corresponding Author : geetha@upm.edu.my
} 